\title{
Trigonella-foenum graecum I. seed mucilage-based mucoadhesive microspheres of diclofenac sodium
}

\begin{abstract}
The study was aimed to discover the properties of Trigonella foenum graecum L. seed mucilage (TFGSM) in the formulation of mucoadhesive microspheres of diclofenac sodium to prolong the residence time at site of absorption. The effects of polymer ratios (TFGSM to sodium alginate) on drug encapsulation efficiency (DEE, \%), mucoadhesivity, swelling index and cumulative drug release (CDR, \%) over $8 \mathrm{~h}$ was investigated. TFGSM-based mucoadhesive microspheres of diclofenac sodium were prepared by employing ionotropic gelation technique. Different polymer ratios of TFGSM and sodium alginate were used to formulate eight formulations (F1 to F8) of TFGSM-based mucoadhesive microspheres of diclofenac sodium and characterized by determining its percentage yield and evaluating DEE (\%), mucoadhesivity, swelling properties and CDR (\%). The DEE (\%) of all eight formulations was within the range of 77.97 to $98.05 \%$ with sustained in vitro release of over $8 \mathrm{~h}$. The in vitro drug release of these microspheres followed controlled release (zero-order). The microspheres possessed good swelling properties and mucoadhesive properties.
\end{abstract}

Keywords: microspheres, trigonella foenum graecum $L$ seed mucilage, mucoadhesion, diclofenac sodium
Volume 7 Issue 2 - 2018

\author{
Senthil Adimoolam, Soudaphone Phonhaxa \\ Department of Dosage Form Design, MAHSA University, \\ Malaysia
}

Correspondence: Senthil Adimoolam, HOD Dosage Form Design, Faculty of Pharmacy, Bandar Saujana Putra, Selangor, Malaysia,Tel +6 0166928506, Email senthil@mahsa.edu.my

Received: February 05, 2018 | Published: March 15, 2018
Abbreviations: NSAIDs, non-steroidal anti-inflammatory drugs; TFGSM, trigonella foenum-graecum seed mucilage; DEE, drug entrapment efficiency

\section{Introduction}

Arthritis is an incurable condition that cannot be cured, especially in chronic cases, by general medication practices but treatments to manage the symptoms and slow the progression are available. Arthritis refers to conditions that involve pain and inflammation of the joints. Patients with arthritis seek treatment, mainly due to the uncomfortable pain that restricts their daily routines. Non-steroidal anti-inflammatory drugs (NSAIDs) are extensively used in the management of arthritisrelated pain. They are effective and available as prescription and non-prescription products. Non-prescription NSAIDs are often used without taking into account the contraindications as they are easily available. ${ }^{1}$ Concerns of the usage of NSAIDs are associated with gastrointestinal (GI) side effects and increased cardiovascular risk.

Diclofenac sodium is a NSAID having anti-inflammatory, analgesic and antipyretic actions. Pharmacokinetically, it rapidly metabolizes due to its relatively short half-life of 1-2 $\mathrm{h}$ and has an absolute bioavailability of $55 \%$ when administered orally due to first pass metabolism effect. ${ }^{3,4}$ Patients require multiple dosing regimens resulting in reduced compliance and poor therapeutic effect significantly. Concerns of the long term usage of diclofenac sodium, like most NSAIDs, is associated with GI problems such as peptic ulcers and GI bleeding along with risks of cardiovascular adverse effects. These concerns of GI side effects has generated more researches with the aim of overcoming the side effect and improve therapeutic effects of diclofenac sodium, leading to development of various conventional oral formulations of diclofenac sodium. Microsphere drug delivery is a multi-particulate system, has been researched to improved absorption and reduces GI irritation that uniformly spreads at site of absorption which is usually formulated using polymers. However, polymeric microspheres have short residence time at site of absorption leading to the development of mucoadhesive microspheres by incorporating mucoadhesive polymers that allows the microspheres to adhere to the mucus tissue and thus, prolong the residence time. ${ }^{6}$

Trigonella foenum-graecum L. seed mucilage (TFGSM) has been investigated as a mucoadhesive polymer. It is originally isolated from Trigonella foenum-gracum $L$. seeds which contains significant amount of mucilage. It is pharmaceutically investigated as binding agent, ${ }^{7}$ disintegrating agent, ${ }^{8}$ and mucoadhesive gelling agent. ${ }^{9}$ However, only a few investigations used Trigonella foenum-graecum seed mucilage (TFGSM) as mucoadhesive excipient in the formulation of mucoadhesive polymer-based microsphere and none of studies used diclofenac sodium as the active ingredient in the development of TFGSM-based mucoadhesive microspheres. In the development of TFGSM-base mucoadhesive microsphere various studies aim to improve mucoadhesive property to extend the residence time at site of absorption as well as to achieve drug encapsulation efficiency and drug release that is within the desired ranged.

The formulation of mucoadhesive polymer-based microspheres using ionotropic gelation techniques is well established. ${ }^{10}$ By using this ionotropic gelation technique allows the polymers to be physically cross-linked to form microspheres and avoiding the utilization of expensive and toxic reagents. ${ }^{11}$ This technique requires counter ions to form the cross-linking.

In previous studies, TFGSM demonstrates promising mucoadhesive properties and is easily available and inexpensive. It offers a new alternative of plant-based mucoadhesive polymer to be used in the formulation of mucoadhesive microspheres. To best of knowledge, no studies have formulated TFGSM-based mucoadhesive microspheres containing diclofenac sodium. It has the potential to 
prolong the residence time at the absorption site, thereby, eliminating frequent dosing and improve patient compliance resulting in improve therapeutic effects. It is beneficial, especially to patients that require long term use of diclofenac sodium such as patients with arthritis.

\section{Materials and methods}

The diclofenac sodium salt was purchased from Sigma Aldrich chemicals Ltd, sodium alginate and all other chemicals were purchased from Sigma Aldrich chemicals Ltd. All the solvents and chemicals used in this study were of analytical-reagent grade. Deionized double distilled water was used throughout the study for any preparation and formulations.

\section{Isolation of TFGSM from trigonella foenum-graecum $L$. seeds}

Trigonella foenum-graecum L. seeds $(200 \mathrm{~g})$ were soaked in 1.51 of distilled water for $24 \mathrm{~h}$ and boiled using water bath until the preparation of slurry is obtained. The slurry were allowed to cool down and kept in refrigerator overnight to settle out undissolved materials. The upper clear solution was gradually poured and concentrated at $60^{\circ} \mathrm{C}$ using water bath to one-third of its original volume. The solution was allowed to cool down and transferred into three times the volume of acetone with continuous stirring. The precipitate was washed repeatedly with acetone and dried at room temperature for $24 \mathrm{~h}$. The isolated material was passed though sieve number 80 and stored in desiccators until further use. ${ }^{12}$

\section{Evaluation of TFGSM}

Solubility: Solubility of TFGSM was performed by dissolving in distilled water and organic solvents such as chloroform and methanol. ${ }^{13,14}$

Swelling index: TFGSM $1 \mathrm{~g}$ was transferred into a $25 \mathrm{ml}$ glass stoppered measuring cylinder and $25 \mathrm{ml}$ of distilled water was added and shaken vigorously every $10 \mathrm{~min}$ for $1 \mathrm{~h}$ and will be allowed to stand for $24 \mathrm{~h}$. The volume occupied by the TFGSM was measured. The process was repeated three times and the swelling index was calculated from the mean of three readings. ${ }^{15,16}$

Loss on drying: Loss on drying was determined by weighing $1 \mathrm{~g}$ of TFGSM and heated at $105^{\circ} \mathrm{C}$ until constant weight is achieved using a hot air oven. ${ }^{17}$ The percentage of moisture loss on drying was calculated using the following formula:

$$
\text { Loss on drying }(\%)=\frac{\text { Weight of water in sample }}{\text { Weight of dry sample }} \times 100
$$

pH: TFGSM $5 \mathrm{~g}$ with $20 \mathrm{ml}$ of distilled water and stirred for $5 \mathrm{~min} . \mathrm{pH}$ of the resulting TFGSM mixture was determined using a calibrated digital $\mathrm{pH}$ meter (Hanna instruments $\mathrm{pH} 211$ microprocessor-based bench $\mathrm{pH}$ meter, USA). ${ }^{17}$

Bulk density: Bulk density was determined by adding pre-weighed amounts of TFGSM into a graduated cylinder and the volume was recorded. The powders were introduced to a tapping in a bulk density apparatus until a constant volume is an achieved..$^{18}$ The tapped density was determined as the ratio of the sample weight to the final sample volume. ${ }^{19}$
Percentage yield: The yield of the TFGSM was calculated using the formula below:

$$
\text { Yield }(\%)=\frac{\text { Amount of TFGSM achieved }(g)}{\text { Amount of TFG seed }(g)} \times 100
$$

\section{Preparation of TFGSM-based mucoadhesive microspheres of diclofenac sodium}

Sodium alginate and TFGSM was weighed and separately dissolved in purified water and mixed to form a polymer dispersion mixture. Diclofenac sodium was added to the polymer dispersion mixture and mixed thoroughly. The resulting polymer-drug dispersion mixture was added drop-wise via a syringe with a needle (No.23) into calcium chloride $(10 \% \mathrm{w} / \mathrm{v})$ solution. The added droplet was retained in the calcium chloride solution for $15 \mathrm{~min}$ to complete the reaction and to produce spherical microspheres. Microsphere was collected by decantation and washed with water and dried overnight at room temperature (Table 1). ${ }^{20}$

\section{Evaluation of TFGSM-based microspheres of diclofenac sodium}

\section{Percentage yield}

The percentage yield of the formulated microspheres was determined using the equation below:

$$
\text { Yield }(\%)=\frac{\text { Amount of microspheres prepared }}{\text { Amount of drug }+ \text { polymer taken }} \times 100
$$

\section{Determination of DEE}

$100 \mathrm{mg}$ of microspheres was crushed and transferred in a $250 \mathrm{ml}$ volumetric flask and the volume was made up to $250 \mathrm{ml}$ using phosphate buffer, $\mathrm{pH} 7.4$ and kept for $24 \mathrm{~h}$ with occasionally shaking at $37 \pm 0.5^{\circ} \mathrm{C}$. After the specified time, the mixture was stirred at $500 \mathrm{rpm}$ for $20 \mathrm{~min}$ using magnetic stirrer. The polymer remaining after disintegration of microspheres was removed by filtering through Whatman filter paper (No. 40). ${ }^{12}$

The drug content in the filtrate was determined using a UV spectrophotometer at $276 \mathrm{~nm}$ against appropriate blank. The DEE (\%) of these prepared microspheres was calculated by the following formula:

$$
\text { DEE }(\%)=\frac{\text { Actual drug content in microspheres }}{\text { Theoretical drug content in microspheres }} \times 100
$$

Table I Formulations of TFGSM-based mucoadhesive microspheres of diclofenac sodium

\begin{tabular}{llll}
\hline Batches & $\begin{array}{l}\text { Diclofenac } \\
\text { sodium }(\mathrm{g})\end{array}$ & TFGSM $\mathbf{( g )}$ & $\begin{array}{l}\text { Sodium } \\
\text { alginate } \\
\mathbf{( g )}\end{array}$ \\
\hline FI & $\mathrm{I}$ & 0.5 & 0.5 \\
F2 & $\mathrm{I}$ & $\mathrm{I}$ & 0.5 \\
F3 & $\mathrm{I}$ & $\mathrm{I} .5$ & 0.5 \\
F4 & $\mathrm{I}$ & 2 & 0.5 \\
F5 & $\mathrm{I}$ & 0.5 & $\mathrm{I}$ \\
F6 & $\mathrm{I}$ & $\mathrm{I}$ & $\mathrm{I}$ \\
F7 & $\mathrm{I}$ & $\mathrm{I} .5$ & $\mathrm{I}$ \\
F8 & $\mathrm{I}$ & 2 & $\mathrm{I}$
\end{tabular}




\section{In vitro drug release studies}

The dissolution rate of microspheres was tested using dissolution apparatus, rotating basket method with two different $\mathrm{pH}$ values, $\mathrm{pH}$ 1.2 and $\mathrm{pH}$ 7.4. The basket was covered with 100 mesh nylon cloth to prevent the escape of the microspheres. The dissolution rates will be measured at $37 \pm 1^{\circ} \mathrm{C}, 50 \mathrm{rpm}$ speed. A weighed quantity of microspheres containing diclofenac sodium $(100 \mathrm{mg})$ was added to $900 \mathrm{ml}$ of $0.1 \mathrm{~N} \mathrm{HCl}, \mathrm{pH} 1.2$. The test was carried out for $2 \mathrm{~h}$ and then continued in phosphate buffer $(\mathrm{pH} 7.4)$ for next $8 \mathrm{~h} .5 \mathrm{ml}$ of aliquots was collected at regular time intervals and the same amount of fresh dissolution medium was replaced into dissolution vessel to maintain the sink condition throughout the experiment. The collect aliquots was filtered and diluted to determine the absorbance using UV spectrophotometer at $276 \mathrm{~nm}$ against appropriate blank. ${ }^{12}$

\section{Swelling study}

The swelling behaviour of the TFGSM-based mucoadhesive microspheres of diclofenac sodium was evaluated by swelling index test. ${ }^{15}$ Swelling index test of TFGSM-based mucoadhesive microspheres of diclofenac sodium was performed in $0.1 \mathrm{~N} \mathrm{HCl}$ $(\mathrm{pH}$ 1.2) and phosphate buffer ( $\mathrm{pH} 7.4)$ aqueous mediums. 100mg of microspheres was placed in the beaker of dissolution apparatus containing $500 \mathrm{ml}$ of respective media. The experiment was carried out at $37 \pm 1{ }^{\circ} \mathrm{C}$ under 50rpm paddle speed. The swelled microspheres was removed at predetermined time interval and weighed after drying the surface by using tissue paper.

Swelling index was determined using the following formula: Swelling index $(\%)=\frac{\text { Weight after swelling }- \text { Dryweight }}{\text { Dryweight of beads }} \times 100$

\section{Ex vivo mucoadhesion testing}

The mucoadhesivity of TFGSM-based mucoadhesive microspheres of diclofenac sodium was evaluated by ex vivo wash-off method. A $1-\mathrm{cm}$ by $1-\mathrm{cm}$ piece of rat stomach mucosa was tied onto a glass slide (3-inch by 1-inch) using thread. Exactly 50 microspheres was placed onto wet tissue specimen and the prepared slide was hung onto the groove of disintegration test apparatus. The tissue specimen was given a regularly up and down movement in a breaker containing $900 \mathrm{ml}$ of $0.1 \mathrm{~N} \mathrm{HCl}(\mathrm{pH} 1.2)$ and phosphate buffer (pH 7.4) separately at $37 \pm 0.5^{\circ} \mathrm{C}$. At the end of time interval, the number of microspheres that remained attached to the stomach mucosa was recorded. ${ }^{21}$

\section{In vitro drug release kinetics and mechanism}

To determine the drug release kinetic and mechanism for the formulated TFGSM-based mucoadhesive microspheres of diclofenac sodium, the in vitro data was evaluated to find a suitable mathematical model to fit the in vitro release behaviour. The following mathematic models were evaluated to determine the drug release per unit time: zero order and first order. Higuchi and Korsmeyer-peppas model was used to evaluate the mechanism of drug release. ${ }^{12}$

Zero order

$$
Q=k t+Q_{0}
$$

Where, $\mathrm{Q}$ represents the drug release amount in time $\mathrm{t}$ and $\mathrm{Q}_{0}$ is the start value of $\mathrm{Q} ; \mathrm{k}$ is the rate constant.
First-order model

$$
Q=Q_{0} e^{k . t}
$$

Where, $\mathrm{Q}$ represents the drug released amount in time $\mathrm{t}$ and $\mathrm{Q}_{0}$ is the start value of $\mathrm{Q} ; \mathrm{k}$ is the rate constant.

Hugachi model

$$
Q=k t^{0.5}
$$

Where, $\mathrm{Q}$ represents the drug released amount in time $\mathrm{t}$ and $\mathrm{k}$ is the rate constant.

Korsmeyer-Peppas model

$$
Q=k t^{n}
$$

Where, Q represents the drug releases amount in time $\mathrm{t}, \mathrm{k}$ is the rate constant and $\mathrm{n}$ is the release exponent, indicative of drug release mechanism.

These models were compared by calculating the squared correlation coefficient $\left(\mathrm{R}^{2}\right)^{22,23}$ Following, the Korsmeyer-Peppas model was used in the in vitro release pattern evaluation of the formulations to distinguish between completing release mechanisms: Fickian release (diffusion-controlled release), non-Fickian release (anomalous transport) and case-II transport (relaxation-controlled release). When $\mathrm{n}$ is $\leq 0.43$, it is Fickian release. The $\mathrm{n}$ value between 0.43 and 0.85 is defined as non-Fickian release. When, $\mathrm{n}$ value is $\geq 0.85$, it is case-II transport. ${ }^{24}$

\section{Fourier transform-infrared (FTIR) spectroscopy}

Samples were powdered and analysed using a Fourier transforminfrared (FTIR) spectroscopy (Perkin Elmer Spectrum two, USA). Small amount of sample powders was placed on the sample area. Spectral scanning was taken in wave length between 4000 and $400 \mathrm{~cm}^{-}$ 1 .

\section{Results and discussion}

The solubility of diclofenac sodium in distilled water at room temperature was $2.86 \mu \mathrm{g} / \mathrm{ml}$. Melting point was determined using open capillary method. The average melting point of diclofenac sodium was $288.66^{\circ} \mathrm{C}$, meeting the specification as stated in British Pharmacopoeia that the range of diclofenac sodium should fall in between $288-290^{\circ} \mathrm{C}$. The standard curve of diclofenac sodium was determined in $0.1 \mathrm{~N} \mathrm{HCl}$ $(\mathrm{pH} 1.2)$ and phosphate buffer $(\mathrm{pH} 7.4)$ at $276 \mathrm{~nm}$. The graph was plotted with concentration as $\mathrm{x}$-axis and absorbance on the y-axis. The plotted graphed followed the Beer-Lambert's law in the concentration of $10-60 \mu \mathrm{g} / \mathrm{mL}$ with a regression correlation $\left(\mathrm{R}^{2}\right)$ of 0.97502 in $0.1 \mathrm{~N}$ $\mathrm{HCl}$ and 0.98727 in phosphate buffer that are approximately close to 1 indicating good linearity in this range of concentration..$^{25}$

The yield of TFGSM isolated from Trigonella foenum-graecum $L$. seeds obtained was less $(3.17 \% \mathrm{w} / \mathrm{w})$ when compared to that of previous isolation performed by other researchers having a higher yield $(17.36 \% \mathrm{w} / \mathrm{w}) .{ }^{12}$ Various tests was performed to evaluate the physicochemical properties and to understand the properties of the TFGSM powder obtained from the isolation of Trigonella foenumgraecum L. seed. Results of physicochemical characteristics of TFGSM powder are shown in Table 2. 
Table 2 Physicochemical characteristics of TFGSM powder

\begin{tabular}{|c|c|}
\hline Tests & Results \\
\hline \multicolumn{2}{|c|}{ Organoleptic Characteristics } \\
\hline Colour & Light brown powder \\
\hline Odour & Odourless \\
\hline \multicolumn{2}{|l|}{ Solubility } \\
\hline Distilled water & $\begin{array}{l}\text { Swells in contact with water and } \\
\text { forms tacky mass. }\end{array}$ \\
\hline Methanol & Insoluble \\
\hline Chloroform & Insoluble \\
\hline \multicolumn{2}{|l|}{ Swelling index } \\
\hline Method I & $30 \mathrm{ml}$ \\
\hline Method II & 735 \\
\hline Loss on drying & $0.46 g$ \\
\hline $\mathrm{pH}$ & 6.23 \\
\hline Bulk density & $0.60 \mathrm{~g} / \mathrm{ml}$ \\
\hline Tap density & $0.69 \mathrm{~g} / \mathrm{ml}$ \\
\hline Yield & $3.17 \%$ \\
\hline of & IFGSM-based \\
\hline
\end{tabular}

The microspheres were prepared by ionotropic gelation technique using TFGSM and sodium alginate as the mucoadhesive agent and calcium chloride as the cross-linker. The polymeric solution of TFGSM, sodium alginate and diclofenac sodium were added dropwised into the calcium chloride solution using a syringe (No.23) forming the microspheres. Sodium alginate is a negatively charge polymer that allows the reaction with positively charge ions present in the calcium chloride solution resulting in the formation of microsphere. ${ }^{23}$ The microspheres general appearance was spherical with rough surfaces and light brown in colour due to the original pigmentation colour of the TFGSM.

It was found that in most formulations when the polymer ratio of the formulation increases the percentage yield also increases. However, low percentage yield can be seen even with high polymer ratio, this might be occurred due to blockage of needle, wastage of the drug-polymer solution and loss during transferring and washing. The percentage yield ranged from 87.40 to $97.80 \%$. The DEE (\%) of F1 to F8 range of 77.97 to $98.05 \%$ was shown in Table 3. It was found that the DEE (\%) was influenced by the increasing polymer ratio of sodium alginate and TFGSM. As the viscosity of the polymeric solution increased, the DEE (\%) decreased. This might be due the increasing TFGSM resulting in a viscous polymeric solution which might have prevented the drug release to the calcium chloride solution. From all eight formulations, F6 was found to have the highest DEE (\%) of 98.05 .

Table 3 Drug encapsulation efficiency of FI to F8

\begin{tabular}{ll}
\hline Formulation & \%DEE \\
\hline FI & 96.87 \\
F2 & 95.69 \\
F3 & 94.51 \\
F4 & 92.15 \\
F5 & 95.69 \\
F6 & 98.05 \\
F7 & 81.51 \\
F8 & 77.97 \\
\hline
\end{tabular}

The in vitro dissolution studies of all eight formulations (F1 to F8) of TFGSM-based mucoadhesive microspheres of diclofenac sodium using a dissolution tester USP (Electrolab, TDT-08L), basket method in $900 \mathrm{ml}$ of $\mathrm{pH} 1.2$ for $2 \mathrm{~h}$ and $\mathrm{pH} 7.4$ for $8 \mathrm{~h}$ at $37^{\circ} \mathrm{C}$ and $50 \mathrm{rmp}$. At each time interval, $10 \mathrm{ml}$ of each formulation was taken and UV absorbance was recorded. The formulations exhibits a percentage cumulative drug release (CDR, \%) in the range of $88.50 \%$ to $97.70 \%$ and was found that in formulations with decreasing ratio of TFGSM was higher. It was found that diclofenac sodium release from these microspheres in $\mathrm{pH} 1.2$ was slow, less than $15.90 \%$ after $2 \mathrm{~h}$. This event might be due to shrinkage of alginate at acidic $\mathrm{pH}$ as alginate is known to be $\mathrm{pH}$ sensitive. ${ }^{23,24}$ In comparison, the drug release was observed to be more rapid in phosphate buffer, due to higher swelling rate of these microspheres in phosphate buffer. F6 showed the highest CDR (\%) of $98.50 \%$. The in vitro drug release of all formulations F1 to F8 is shown in Figure 1.

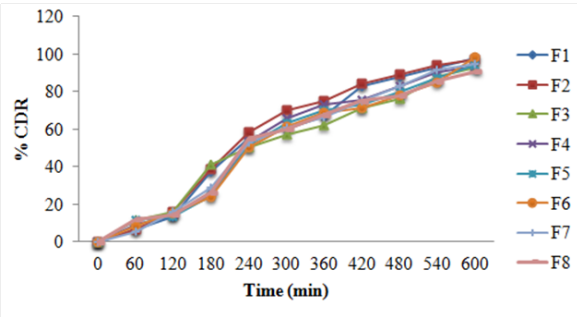

Figure I In vitro drug release of FI to F8.

The swelling pattern of F1 to F8 ionotropically TFGSM-based mucoadhesive microspheres of diclofenac sodium were evaluated in $0.1 \mathrm{~N} \mathrm{HCl}$ and phosphate buffer. The swelling patterns of these microspheres in both medium were shown in Figure 2 \& Figure 3. It was observed that as the polymer concentration increases, the microspheres swells more. Initially, the extent of swelling of these TFGSM-based mucoadhesive microspheres of diclofenac sodium was lower in acidic medium compared with that of in phosphate buffer. This resulted due to shrinkage of alginate in acidic $\mathrm{pH} .{ }^{23}$ Maximum swelling of the microspheres was observed at $2-3 \mathrm{~h}$ in phosphate buffer and followed by erosion and dissolution. It was reported that phosphate ions present in the phosphate buffer can behave as calcium sequestrants, allowing further swelling of calcium alginate microspheres. This swelling pattern of TFGSM-based mucoadhesive microspheres of diclofenac sodium in phosphate buffer resulted by the exchange of ions between calcium ions present in phosphate buffer with the influence of calcium sequestrant phosphate ions. This phenomenon might result in disaggregation of the TFGSM and alginate matrix structure resulting in matrix erosion and dissolution of the swollen microspheres. ${ }^{26,27}$ These indicates that these TFGSMbased mucoadhesive microspheres of diclofenac sodium may show slight swelling in the stomach as they subsequently move to upper intestine, where the diclofenac sodium is to be absorbed, and these microspheres continue to swell more.

The ex-vivo wash-off test was performed using rat stomach mucosa for evaluating the mucoadhesive property of TFGSMbased mucoadhesive microspheres of diclofenac sodium for F6 was performed at stomach $\mathrm{pH}$ for $8 \mathrm{~h}$ using disintegration apparatus. The percentage of microspheres remained adhered to the rat stomach mucosal tissue was $56 \%$ over $8 \mathrm{~h}$, shown in Figure 4 . The decreasing trend of the mucoadhesion of TFGSM-based mucoadhesive microspheres of diclofenac sodium in phosphate buffer might 
be occurred due to erosion of calcium ion. Therefore, the results of the wash-off test indicated that TFGSM-based mucoadhesive microspheres of diclofenac sodium possessed good mucoadhesivity.

TFGSM-based mucoadhesive microspheres of diclofenac sodium F6 was evaluated mathematically for its drug release kinetics model like zero order, first order, Higguchi and Korsmeyer-Peppas models. The $\mathrm{R}^{2}$ of these models were determined and compared. The $\mathrm{R}^{2}$ of TFGSM-based mucoadhesive microspheres of diclofenac sodium F6 were compared, it was found to follow zero-order model $\left(\mathrm{R}^{2}=0.966\right)$ as the best-fit model over a period of $8 \mathrm{~h}$ amongst others. This was also observed to be closest to Higuchi model $\left(\mathrm{R}^{2} 0.964\right)$. Finally, the best fitting of the zero-order model indicates that the drug release from F6 microspheres followed controlled-release pattern. The value of diffusional exponent (n) determined from Korsmeyer-Peppas model $(\mathrm{n}=0.188)$, indicating the drug release from these microspheres followed Fickian mechanism. Fickian diffusion refers to the solute transport process in which the polymer relaxation time is much greater

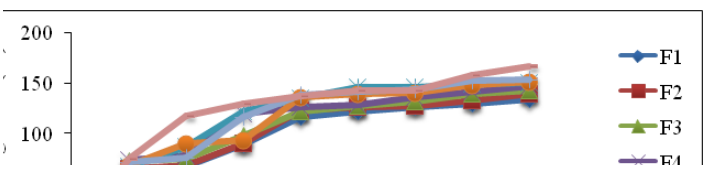

Figure 2 Swelling behaviour in $0.1 \mathrm{~N} \mathrm{HCl}(\mathrm{pH}$ I.2).

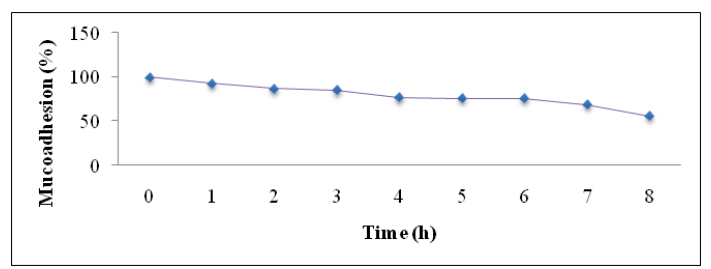

Figure 4 Result of ex-vivo wash-off test for (F6) in phosphate buffer.

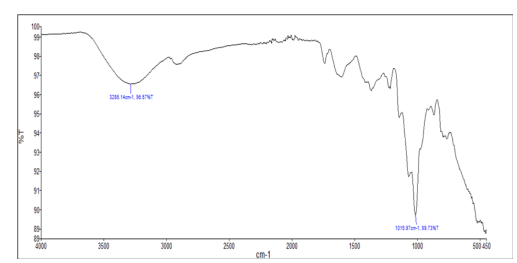

Figure 6 FTIR spectrum of TFGSM.

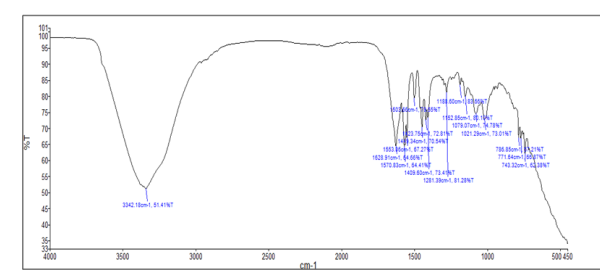

Figure 8 FTIR spectrum of TFGSM-based microspheres of diclofenac sodium. than the characteristic solvent diffusion time. ${ }^{28}$

The FTIR spectras of diclofenac sodium, TFGSM, sodium alginate and TFGSM-sodium algintate microspheres containing diclofenac sodium are shown in Figure 5-8. The FTIR spectrum of diclofenac sodium showed the bands around 611.70, 748.06, 1452.30 and $1603.84 \mathrm{~cm}^{-1}$, which are due to the bending of $=\mathrm{C}-\mathrm{H}$ and stretching of $\mathrm{C}=\mathrm{C}$. In FTIR spectrum of TFGSM showed absorption characteristic peaks of ether linkage C-O at 1015.97 and aromatic stretching of C-H at $3286.14 \mathrm{~cm}^{-1}$. The FTIR spectrum of sodium alginate showed peaks at $1024.75,1407.82$ and 1594.77 , indicating the presence of an ether linkage $\mathrm{C}-\mathrm{O}$, $-\mathrm{C}-\mathrm{H}$ bending alkenes and $\mathrm{C}=\mathrm{C}$ aromatic stretching. Lastly, the FTIR spectrum for TFGSM-sodium alginate microspheres containing diclofenac sodium, showed several characteristics of peaks of diclofenac sodium, sodium alginate and TFGSM were present and showed no shifting of these peaks, suggesting there were no interactions between drug and excipient used (sodium alginate and TFGSM).

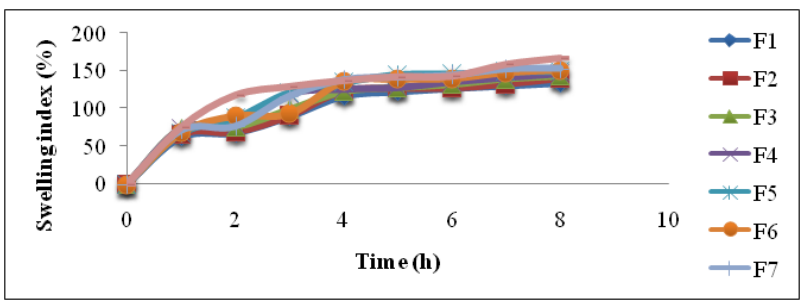

Figure 3 Swelling behaviour in phosphate buffer.

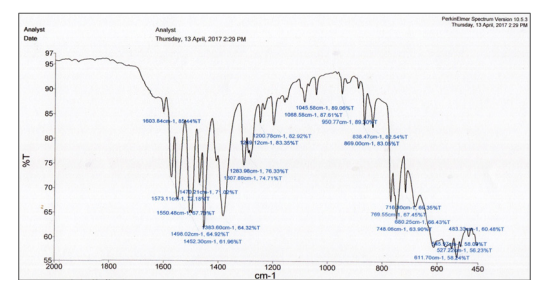

Figure 5 FTIR spectrum of diclofenac sodium.

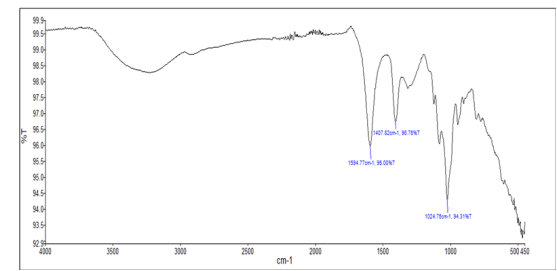

Figure 7 FTIR spectrum of sodium alginate.

\section{Conclusion}

TFGSM-based mucoadhesive microsphere of diclofenac sodium by ionotropic gelation techniques were successfully formulated and evaluated. The study shows that the TFGSM-based microspheres formulated were able to prolong gastric residence time by swelling slowly in stomach and adhering to the stomach mucosa which then will subsequently move to the intestine and swells more allowing the release of drug. Thus, TFGSM serves as a potential mucoadhesive excipient in the formulation of controlled-release mucoadhesive microsphere. 


\section{Acknowledgements}

None.

\section{Conflict of interest}

The author declares there is no conflict of interest.

\section{References}

1. Vonkeman HE, Fernandes RW, van der Palen J, et al. Proton-pump inhibitors are associated with a reduced risk for bleeding and perforated gastroduodenal ulcers attributable to non-steroidal anti-inflammatory drugs: a nested case-control study. Arthritis Res Thera. 2007;9(3):R52.

2. Al-Saeed A. Gastrointestinal and cardiovascular risk of nonsteroidal antiinflammatory drugs. Oman Med J. 2011;26(6):385-391.

3. Brogden RN, Heel RC, Pakes GE, et al. Diclofenac sodium: A review of its pharmacological properties and therapeutic use in rheumatic diseases and pain of varying origin. Drugs. 1980;20(1):24-48.

4. Small R. Diclofenac sodium. Clin pharm. 1989;8(8):545-558.

5. Gan TJ. Diclofenac: an update on its mechanism of action and safety profile. Curr Med Res Opin.2010;26(7):1715-1731.

6. Sam M, Gayathri D, Prasanth V, et al. NSAIDs as microspheres. Internet J Pharmacol. 2007;6(1):233-239.

7. Arsul VA, Lahoti DP. Natural polysaccharides as pharmaceuticals excipients. World J Pharm Res. 2004;3(2):3776.

8. Kumar R, Patil S, Patil MB, et al. Isolation and evaluation of disintegrant properties of fenugreek seed mucilage. Int $J$ Pharm Tech Res. 2009;1(4):982-996.

9. Datta R, Bandyopadhyay AK. Development of a new nasal drug delivery system of diazepam with natural mucoadhesive agent from Trigonella foenum-graecum L. J Sci Ind Res. 2005;64:973-977.

10. Patil JS, Kamalapur MV, Marapur SC, et al. Ionotropic gelation and polyelectrolyte complexation: the novel techniques to design hydroge particulate sustained, modulated drug delivery system: a review. Digest Nanomat Biost. 2010;5(1):241-248.

11. Patil P, Chavanke D, Wagh M. A review on ionotropic gelation method: novel approach for controlled gastroretentive gelispheres. Int $J$ Phar Pharm Sci. 2012;4(4):27-32.

12. Nayak AK, Pal D, Pradhan J, Hasnain MS. Fenugreek seed mucilagealginate mucoadhesive beads of metformin $\mathrm{HCl}$ : Design, optimization and evaluation. Int J Bio Macromol. 2013;54:144-154.

13. Modi A, Tayade PA. Comparative solubility enhancement profile of Valdecoxib with different solubilization approaches. Indian J Pharm Sci. 2007;69(2):274-278.
14. European Pharmacopoeia. 5th ed. Council of Europe, France. 2015.

15. Naveed S, Qamar F. UV spectrophotometric assay of diclofenac sodium available brands. Int Inno Pharm Bio Sci. 2014;1(3):92-96.

16. Bal T, Murthy PN, Sengupta S. Isolation and analytical studies of mucilage obtained from the seeds of Dillenia indica (family dilleniaceae) by use of various analytical techniques. Asian J Pharm Cli Res. 2012;5(33):65-67.

17. Patel DM, Prajapati G, Patel NM. Seed mucilage from Ocimum americanum Linn. as disintegrant in tablets: Separation and evaluation. Ind J Pharm Sci. 2007;3(69):431.

18. Pabon CV, Frutos P, Lastres JL, et al. In vitro study of mixed controlled release matrix tablets containing HPMC and polyamide. Drug Dev Ind Phar. 1992;18(20): 2163-71.

19. Kumar D, Singhal A, Bansal S. Extraction, isolation and evaluation Trigonella foenum-graecum as mucoadhesive agent for nasal gel durg delivery. Journal of Nepal Pharmaceutical Association. 2014;27(1).

20. Erdandi C. Preparations of microspheres of diclofenac sodium by ionotrpic geletion technique. Int J Pha Pharm Sci. 2013;5(1):228-231.

21. Pal D, Nayak AK. Development, Optimization, and anti-diabetic activity of gliclazide-loaded alginate-methylcellulose mucoadhesive microcapsules. AAPS Pharm Sci Tec. 2011;12(4):1431-1441.

22. Lim F, Moss RD. Microencapsulation of living cells and tissues. J Pharm Sci. 1981;70(4):351-354.

23. Jui Jung Chuang, Huang Yu Ya, Lo Szu-Hsuan, et al. (2017) Effects of pH on the shape of alginate particles and its release behavior. Int J Pol Sci. 2017;(1):1-9.

24. Mukhopadhyay P, Sarkar K, Soam S, et al. Formulation of pH-responsive carboxy methylcellulose, chitosan and alginate beads for the oral delivery of insulin. J App Pol Sci. 2017;129(2):835-845.

25. Ravi sankar S. Textbook of pharmaceutical analysis. 2 edn. 1998;1-13.

26. Gaudio P, Colombo P, Colombo G, et al. Mechanism of formation and disintegration of alginate beads obtained by prilling. Int $J$ Pharma. 2005;302(1-2):1-9.

27. Ostberg T, Graffner C. Calcium alginate matrices or oral multiple unit administration: III influence of calcium concentration, amount of drug added and alginate characteristics on drug release. International Journal of Pharmaceutics. 1194;111(3):271-287.

28. Grassi M, Grassi G. Mathemathical modelling and controlled drug delivery: matrix systems. Current Drug Delivery. 2005;2(1):97-116. 